\title{
The Unitarity Triangle Fit in the Standard Model and Hadronic Parameters from Lattice QCD: A Reappraisal after the Measurements of $\Delta m_{s}$ and $B R\left(B \rightarrow \tau \nu_{\tau}\right)$
} $\mathrm{UT}_{\text {fit }}$

UTfit Collaboration :

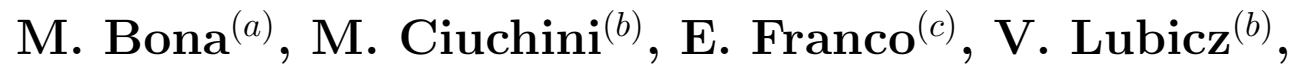

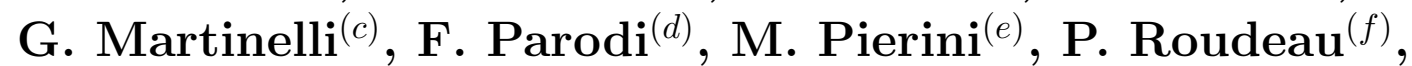 \\ C. Schiavi ${ }^{(d)}$, L. Silvestrini ${ }^{(c)}$, A. Stocchi ${ }^{(f)}$ and V. Vagnoni ${ }^{(g)}$ \\ (a) Laboratoire d'Annecy-le-Vieux de Physique des Particules, \\ LAPP, IN2P3/CNRS, Université de Savoie, France \\ (b) Dip. di Fisica, Università di Roma Tre and INFN, Sez. di Roma III, \\ Via della Vasca Navale 84, I-00146 Roma, Italy \\ (c) Dip. di Fisica, Università di Roma "La Sapienza" and INFN, Sez. di Roma, \\ Piazzale A. Moro 2, 00185 Roma, Italy \\ (d) Dip. di Fisica, Università di Genova and INFN, \\ Via Dodecaneso 33, 16146 Genova, Italy \\ (e) Department of Physics, University of Wisconsin, \\ Madison, WI 53706, USA \\ (f) Laboratoire de l'Accélérateur Linéaire, \\ IN2P3-CNRS et Université de Paris-Sud, BP 34, F-91898 Orsay Cedex, France \\ ${ }^{(g)}$ INFN, Sez. di Bologna, \\ Via Irnerio 46, I-40126 Bologna, Italy
}

\begin{abstract}
The recent measurements of the $B_{s}^{0}$ meson mixing amplitude by CDF and of the leptonic branching fraction $B R\left(B \rightarrow \tau \nu_{\tau}\right)$ by Belle call for an upgraded analysis of the Unitarity Triangle in the Standard Model. Besides improving the previous constraints on the parameters of the CKM matrix, these new measurements, combined with the recent determinations of the angles $\alpha, \beta$ and $\gamma$ from non-leptonic decays, allow, in the Standard Model, a quite accurate extraction of the values of the hadronic matrix elements relevant for $K^{0}-\bar{K}^{0}$ and $B_{s, d^{0}}^{0} \bar{B}_{s, d}^{0}$ mixing and of the leptonic decay constant $f_{B}$. These values, obtained "experimentally", can then be compared with the theoretical predictions, mainly from lattice QCD. In this paper we upgrade the UT fit, we determine from the data the kaon $B$-parameter $\hat{B}_{K}$, the $B^{0}$ mixing amplitude parameters $f_{B_{s}} \hat{B}_{B_{s}}^{1 / 2}$ and $\xi$, the decay constant $f_{B}$, and make a comparison of the obtained values with lattice predictions. We also discuss the different determinations of $V_{u b}$ and show that current data do not favour the value measured in inclusive decays.
\end{abstract}




\section{Introduction}

Lattice QCD (LQCD) played a relevant role in the history the Unitarity Triangle (UT) fit since the very beginning [1-4], allowing predictions of the value of $\sin 2 \beta$ before the advent of direct measurements by Babar and Belle $[5,6]$. At the time when the B factories had not started yet and inclusive measurements of $\left|V_{u b}\right|$ and $\left|V_{c b}\right|$ were rather rough, the "classical" UT analysis for the determination of $\bar{\rho}$ and $\bar{\eta}$ relied on the results of quenched lattice QCD simulations to relate the measured exclusive semileptonic $B$ decays, the $B_{d}^{0}$ $\bar{B}_{d}^{0}$ mixing amplitude, the lower bound on $B_{s}^{0}-\bar{B}_{s}^{0}$ oscillations and CP violation in $K^{0}-\bar{K}^{0}$ mixing to the CKM parameters. In spite of these caveat our prediction of $\sin 2 \beta$ in the years was quite stable, going from $\sin 2 \beta=0.65 \pm 0.12$ in 1995 [1] to $\sin 2 \beta=0.698 \pm 0.066$ in 2000 [4].

A similar situation is true for $\Delta m_{s}$, for which a first precise indirect determination from the other constraints of the UT fit was available since $1997\left([6.5,15.0] \mathrm{ps}^{-1}\right.$ at $68 \%$ probability and $\Delta m_{s}<22 \mathrm{ps}^{-1}$ at $95 \%$ probability) [3]. A compilation of the predictions for $\Delta m_{s}$ by various collaborations as a function of time is shown in Fig. 1. As can be seen from this figure, even in recent years, and despite the improved measurements, in some approaches $[8,10]$ the predicted range was very large (or corresponds only to a lower bound [8]). An upgraded version of our Standard Model "prediction" for $\Delta m_{s}$, obtained from an overall UT fit which makes use of all the latest input values and constraints, is given in the fifth column of Tab. 2. $\Delta m_{s}=(20.9 \pm 2.6) \mathrm{ps}^{-1}$. This is the number and uncertainty to compare with the direct CDF measurement given in eq. (11) below. Besides, in Fig. 2 we also show the compatibility plot for $\Delta m_{s}[9]$.

More recently, we got much more information coming from the determination of the UT angles, obtained by studying non-leptonic decays: the angle $\alpha$ from $B \rightarrow \pi \pi, B \rightarrow \pi \rho$ and $B \rightarrow \rho \rho$ decays [12]; the angle $\gamma$ from $B \rightarrow D^{(*)} K^{(*)}$ decays [13]; $2 \beta+\gamma$ from timedependent asymmetries in $B \rightarrow D^{(*)} \pi(\rho)$ decays [14]; $\cos 2 \beta$ from $B_{d}^{0} \rightarrow J / \psi K_{S}^{* 0}[15]$; $\beta$ from $B \rightarrow D^{0} \pi^{0}[16]$ and, finally, sin $2 \beta$ from the "golden mode" $B_{d}^{0} \rightarrow J / \psi K_{S}$ [17]. In the following we will call the ensemble of these measurements UTangles: they allow a determination of $\bar{\rho}$ and $\bar{\eta}$ independently of the hadronic parameters computed on the lattice. The precision in constraining $\bar{\rho}$ and $\bar{\eta}$ from the UTangles is by now comparable to that obtained from lattice-related constraints, denoted as UTlattice. The latter include, besides the information coming from semileptonic decays, namely $\left|V_{u b}\right| /\left|V_{c b}\right|$, the experimental quantities $\epsilon_{K}, \Delta m_{d}$ and $\Delta m_{s}$.

The recent measurements of the neutral $B_{s}$ meson mixing amplitude by the CDF Collaboration [11], and of the leptonic branching fraction $B R\left(B \rightarrow \tau \nu_{\tau}\right)$ by the Belle Collaboration [18]

$$
\begin{aligned}
\Delta m_{s} & =\left(17.33_{-0.21}^{+0.42} \text { (stat.) } \pm 0.07 \text { (syst.) }\right) \mathrm{ps}^{-1} \quad \mathrm{CDF} \\
B R\left(B \rightarrow \tau \nu_{\tau}\right) & =\left(1.06_{-0.28}^{+0.34} \text { (stat.) }{ }_{-0.16}^{+0.18} \text { (syst.) }\right) \times 10^{-4} \quad \text { Belle }
\end{aligned}
$$

and the additional bounds given respectively by the D0 [19] and BaBar [20] Collaborations, provide further information for the analysis of the Unitarity Triangle in the Standard Model. In this paper, besides improving the determination of the constraints on the parameters of the CKM matrix via the standard UT analysis, we show that the new measurements allow a quite accurate extraction of the values of the hadronic matrix elements relevant for $K^{0}-\bar{K}^{0}$ and $B_{s, d^{-}}^{0} \bar{B}_{s, d}^{0}$ mixing and of the leptonic decay constant $f_{B}$. Assuming that there is no contribution from New Physics, we determine these hadronic quantities 


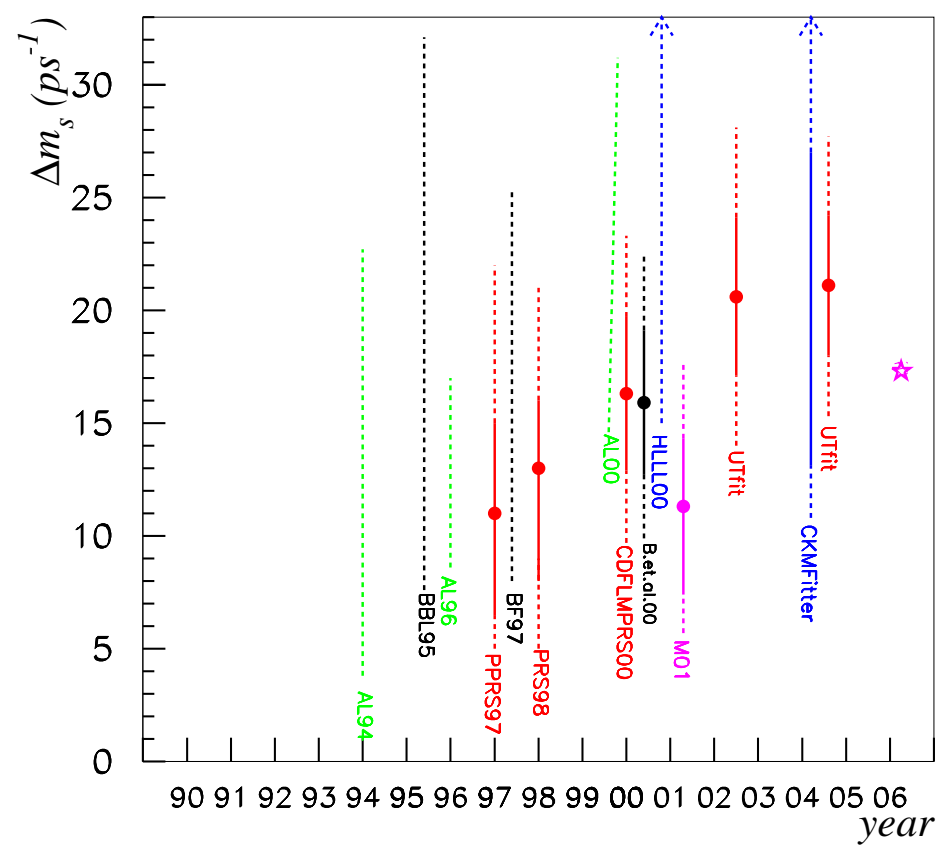

Figure 1: Evolution of the "indirect" determination of $\Delta m_{s}$ over the years. These determinations are given in [3, 4, 7-10]. From left to right, they correspond to the following papers: AL94 (Ali, London), BBL95 (Buchalla,Buras,Lautenbacher), AL96, PPRS97 (Paganini, Parodi, Roudeau, Stocchi), BF97 (Buras,Fleischer), PRS98 (Parodi,Roudeau,Stocchi), AL00, CDFLMPRS00 (Ciuchini et al.), B.et.al.00 (Bargiotti et al.), HLLL00 (Hoecker, Laplace,Lacker,LeDiberder), M01 (Mele), UTFit (Bona et al.). CKMFitter (J.Charles et al.). The full (dotted) lines correspond to the 68\%(95\%) probability regions. The star (for year '06) corresponds to the recent measured value by CDF [11]. The error of the experimental measurement cannot be appreciated with this scale.

from the experimental data and compare them with recent lattice calculations [21,22]. We also discuss the different determinations of $V_{u b}$ and show that there is an indication that the value measured in inclusive decays is not favoured by the data.

\section{Upgraded UTfit Analysis}

In this section we give the results of the upgraded analysis which includes the new measurement of $\Delta m_{s}$ by the CDF Collaboration. This result improves the determination of $\Delta m_{s}$ by LEP, SLD and previous TeVatron analyses [19,23]. Given the uncertainty on the theoretical value of $f_{B}$ and the still relatively large error in the experimental measurement, the effect of $B R\left(B \rightarrow \tau \nu_{\tau}\right)$ on the analysis is negligible at this stage. Indeed by taking from the lattice $f_{B}=(189 \pm 27) \mathrm{MeV}[22]$, one gets $\left|V_{u b}\right|=(41 \pm 9) \times 10^{-4}$ with an error much larger than the uncertainty of determinations from exclusive or inclusive semileptonic decays.

In Tab. 11 we give the value of the upgraded input parameters. In some cases the same quantities, e.g. $\sin 2 \beta$, also appear, with a different central value and uncertainty, 


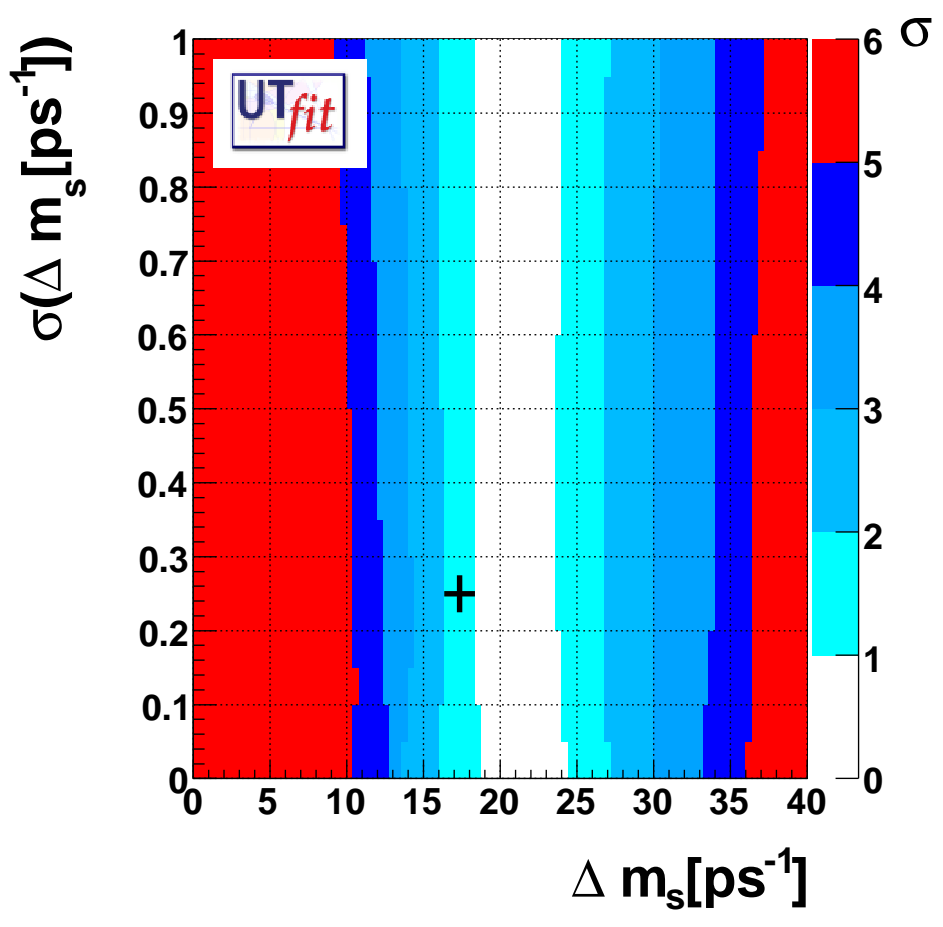

Figure 2: Compatibility plot of the value of $\Delta m_{s}$ measured by CDF, $\Delta m_{s}=$ $\left(17.33_{-0.21}^{+0.42}\right.$ (stat.) \pm 0.07 (syst.)) $\mathrm{ps}^{-1}$ with the upgraded "prediction" from the other constraints of the Standard Model UT fit.

in Tab. 2. where we give the output results of the UT fit. The reason is that the final output values of Tab. 2 are obtained by combining all the available information on a given quantity $[3,4,9]$ : in the case of $\sin 2 \beta$, for example, the information coming from the UTangles and UTlattice measurements.

In Fig. [3] we show the results of the new fit which includes all constraints: $\left|V_{u b}\right| /\left|V_{c b}\right|$, $\Delta m_{d}, \Delta m_{s}, \varepsilon_{K}, \alpha, \beta$, and $\gamma$. In addition in Tab. 2 we present for comparison the values and uncertainties of the relevant quantities for the two cases, UTangles and UTlattice, whereas in the column labelled as "All" we give the results of the analysis including all constraints. ${ }^{1}$

Several observations are important at this point:

- The recent measurement of $\Delta m_{s}$ reduces the uncertainties, although not in a dramatic way.

- If we compare Tab. 1 and Tab. 2 with the corresponding ones of our previous published UT analysis [9], we note that the directly measured value of $\sin 2 \beta$ has decreased from $\sin 2 \beta=0.726$ (37) (old) to $\sin 2 \beta=0.687$ (32) (new). As a consequence, the overlap between the regions of the $\bar{\rho}-\bar{\eta}$ plane, selected by the UTangles with respect to the region selected by the UTlattice, is reduced. This is shown in Fig. 固 where we superimpose the region selected by the UTangles to the $68 \%$ and $95 \%$

\footnotetext{
${ }^{1}$ For further details on the UT analysis of the UTfit Collaboration see refs. [4,9,24]; for the results of the CKMfitter collaboration see [10,25].
} 


\begin{tabular}{|c|c|c|c|}
\hline Parameter & Value & Gaussian $(\sigma)$ & $\begin{array}{l}\text { Uniform } \\
\text { (half-width) }\end{array}$ \\
\hline$\lambda$ & 0.2258 & 0.0014 & - \\
\hline$\left|V_{c b}\right|$ (excl.) & $41.4 \times 10^{-3}$ & $2.1 \times 10^{-3}$ & - \\
\hline$\left|V_{c b}\right|$ (incl.) & $41.6 \times 10^{-3}$ & $0.7 \times 10^{-3}$ & $0.6 \times 10^{-3}$ \\
\hline$\left|V_{u b}\right|($ excl. $)$ & $38.0 \times 10^{-4}$ & $2.7 \times 10^{-4}$ & $4.7 \times 10^{-4}$ \\
\hline$\left|V_{u b}\right|$ (incl.) & $44.5 \times 10^{-4}$ & $2.0 \times 10^{-4}$ & $2.6 \times 10^{-4}$ \\
\hline$\overline{\Delta m_{d}}$ & $0.502 \mathrm{ps}^{-1}$ & $0.006 \mathrm{ps}^{-1}$ & - \\
\hline$\Delta m_{s}$ & $17.35 \mathrm{ps}^{-1}$ & ${ }_{-0.21}^{+0.42} \pm 0.07 \mathrm{ps}^{-1}$ & - \\
\hline$f_{B_{s}} \sqrt{\hat{B}_{B_{s}}}$ & $262 \mathrm{MeV}$ & $35 \mathrm{MeV}$ & - \\
\hline$\xi=\frac{f_{B_{s}} \sqrt{\hat{B}_{B_{s}}}}{f_{B_{d}} \sqrt{\hat{B}_{B_{d}}}}$ & 1.23 & 0.06 & - \\
\hline$\hat{B}_{K}$ & 0.79 & 0.04 & 0.08 \\
\hline$\varepsilon_{K}$ & $2.280 \times 10^{-3}$ & $0.013 \times 10^{-3}$ & - \\
\hline$f_{K}$ & $0.159 \mathrm{GeV}$ & fixed & \\
\hline$\Delta m_{K}$ & $0.5301 \times 10^{-2} \mathrm{ps}^{-1}$ & fixed & \\
\hline$\overline{\sin 2 \beta}$ & 0.687 & 0.032 & - \\
\hline$\overline{\bar{m}}_{t}$ & $168.5 \mathrm{GeV}$ & $4.1 \mathrm{GeV}$ & - \\
\hline $\bar{m}_{b}$ & $4.21 \mathrm{GeV}$ & $0.08 \mathrm{GeV}$ & - \\
\hline $\bar{m}_{c}$ & $1.3 \mathrm{GeV}$ & $0.1 \mathrm{GeV}$ & - \\
\hline$\alpha_{s}\left(M_{Z}\right)$ & 0.119 & 0.003 & - \\
\hline$G_{F}$ & $1.16639 \times 10^{-5} \mathrm{GeV}^{-2}$ & fixed & \\
\hline$m_{W}$ & $80.425 \mathrm{GeV}$ & fixed & \\
\hline$m_{B_{d}^{0}}$ & $5.279 \mathrm{GeV}$ & fixed & \\
\hline$m_{B_{s}^{0}}$ & $5.375 \mathrm{GeV}$ & fixed & \\
\hline$m_{K}^{0}$ & $0.497648 \mathrm{GeV}$ & fixed & \\
\hline
\end{tabular}

Table 1: Values of the relevant input quantities used in the UT fit. The Gaussian and the flat contributions to the uncertainty are given in the third and fourth columns respectively (for details on the statistical treatment see [4]). 


\begin{tabular}{ccccccc}
\hline \hline Parameter & UTangles & UTlattice & All & All $\left[\right.$ no $\left.\Delta m_{s}\right]$ & All $\left[V_{u b}\right.$-excl $]$ & All $\left[V_{u b}\right.$-incl $]$ \\
\hline $\bar{\rho}$ & $0.204 \pm 0.055$ & $0.197 \pm 0.035$ & $0.197 \pm 0.031$ & $0.228 \pm 0.034$ & $0.167 \pm 0.031$ & $0.197 \pm 0.032$ \\
\hline $\bar{\eta}$ & $0.317 \pm 0.025$ & $0.389 \pm 0.025$ & $0.351 \pm 0.020$ & $0.336 \pm 0.021$ & $0.334 \pm 0.018$ & $0.351 \pm 0.020$ \\
\hline$\alpha\left[^{\circ}\right]$ & $100 \pm 8$ & $90.8 \pm 4.9$ & $95.5 \pm 4.8$ & $99.5 \pm 4.5$ & $94.4 \pm 4.6$ & $95.5 \pm 4.9$ \\
\hline$\beta\left[^{\circ}\right]$ & $21.8 \pm 1.3$ & $25.8 \pm 1.4$ & $23.6 \pm 1.0$ & $21.8 \pm 1.3$ & $21.8 \pm 1.1$ & $23.5 \pm 1.0$ \\
\hline $\sin 2 \beta$ & $0.687 \pm 0.032$ & $0.784 \pm 0.032$ & $0.733 \pm 0.024$ & $0.730 \pm 0.023$ & $0.689 \pm 0.028$ & $0.734 \pm 0.024$ \\
\hline $\sin 2 \beta_{s}$ & $0.034 \pm 0.003$ & $0.041 \pm 0.003$ & $0.037 \pm 0.002$ & $0.036 \pm 0.002$ & $0.036 \pm 0.002$ & $0.038 \pm 0.002$ \\
\hline$\left.\gamma^{\circ}\right]$ & $57.4 \pm 8.4$ & $63.0 \pm 4.8$ & $60.6 \pm 4.7$ & $55.8 \pm 5.2$ & $63.5 \pm 4.6$ & $60.7 \pm 4.8$ \\
\hline $\operatorname{Im} \lambda_{\mathrm{t}}\left[10^{-5}\right]$ & $12.6 \pm 1.1$ & $15.3 \pm 0.9$ & $14.1 \pm 0.7$ & $13.7 \pm 0.8$ & $13.3 \pm 0.7$ & $14.2 \pm 0.08$ \\
\hline$\Delta m_{s}\left[\mathrm{ps}^{-1}\right]$ & $20 \pm 5$ & $17.4 \pm 0.3$ & $17.5 \pm 0.3$ & $20.9 \pm 2.6$ & $17.4 \pm 0.3$ & $17.4 \pm 0.3$ \\
\hline$V_{u b}\left[10^{-3}\right]$ & $3.67 \pm 0.24$ & $4.18 \pm 0.20$ & $3.91 \pm 0.14$ & $3.96 \pm 0.14$ & $3.60 \pm 0.17$ & $3.92 \pm 0.16$ \\
\hline$V_{c b}\left[10^{-2}\right]$ & $4.15 \pm 0.07$ & $4.12 \pm 0.07$ & $4.17 \pm 0.06$ & $4.19 \pm 0.06$ & $4.15 \pm 0.06$ & $4.17 \pm 0.06$ \\
\hline$V_{t d}\left[10^{-3}\right]$ & $8.03 \pm 0.57$ & $8.30 \pm 0.31$ & $8.26 \pm 0.31$ & $7.97 \pm 0.34$ & $8.43 \pm 0.28$ & $8.26 \pm 0.32$ \\
\hline$\left|V_{t d} / V_{t s}\right|$ & $0.197 \pm 0.015$ & $0.205 \pm 0.009$ & $0.201 \pm 0.008$ & $0.192 \pm 0.009$ & $0.206 \pm 0.007$ & $0.201 \pm 0.008$ \\
\hline$R_{b}$ & $0.382 \pm 0.024$ & $0.438 \pm 0.023$ & $0.404 \pm 0.015$ & $0.408 \pm 0.015$ & $0.374 \pm 0.018$ & $0.404 \pm 0.016$ \\
\hline$R_{t}$ & $0.856 \pm 0.058$ & $0.891 \pm 0.036$ & $0.875 \pm 0.034$ & $0.841 \pm 0.037$ & $0.897 \pm 0.031$ & $0.875 \pm 0.034$ \\
\hline \hline
\end{tabular}

Table 2: Comparison of determinations of UT parameters from the constraints on the angles $\alpha, \beta$, and $\gamma$ (UTangles) and from lattice-dependent quantities $\left|V_{u b} / V_{c b}\right|, \Delta m_{d}, \Delta m_{s}$, and $\epsilon_{K}$ (UTlattice). We also show the results obtained by using all the constraints together (All), all the constraints except $\Delta m_{s}\left(\mathrm{All}\left[\right.\right.$ no $\left.\left.\Delta m_{s}\right]\right)$, all the constraints except the inclusive $\left|V_{u b}\right|\left(\mathrm{All}\left[V_{u b}\right.\right.$-excl $\left.]\right)$ and all the constraints except the exclusive $\left|V_{u b}\right|$ (All[ $\left[V_{u b}\right.$-incl $\left.]\right)$. For the definition of $R_{b}$ and $R_{t}$ see for example ref. [26], for the definition of $\sin 2 \beta_{s}$ see ref. [27].

probability contours coming from the UTlattice fit. A similar figure with 2004 data would have given a much better agreement. Besides the fact that the measurements are now more precise, the worse agreement is due to i) the lower value of $\sin 2 \beta$ and ii) an important reduction of the quoted uncertainty of the inclusive $\left|V_{u b}\right|$.

- The difference between the results with UTangles and UTlattice is also demonstrated by a comparison of the experimental value, $\sin 2 \beta=0.687(32)$, with the value obtained by using only the UTlattice measurements, $\sin 2 \beta_{\text {UTlattice }}=0.784(32)$.

- $\bar{\eta}$ is also an instructive quantity to visualize the important difference between the UTangles result, $\bar{\eta}_{\text {UTangles }}=0.317 \pm 0.025$ and the $\mathbf{U T}$ lattice case, $\bar{\eta}_{\text {UTlattice }}=0.389 \pm$ 0.025 .

- In order to understand where these differences come from, we have studied the correlation between the value of $\sin 2 \beta_{\text {UTlattice }}$ and $\left|V_{u b}\right|$ with the following results: if we use only the exclusive value of $\left|V_{u b}\right|$, we get $\sin 2 \beta_{\text {UTlattice-excl. }}=0.704(55)$, much closer to $\sin 2 \beta_{\text {UTangles }}=0.687(32)$ whereas if we use only the inclusive value of $\left|V_{u b}\right|$ we obtain $\sin 2 \beta_{\text {UTlattice-incl. }}=0.804(37)$. This implies that there is a strong

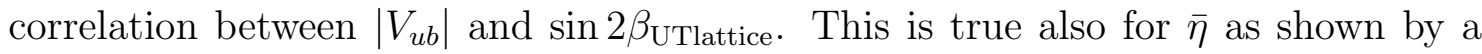
comparison between $\bar{\eta}_{\text {UTlattice-excl. }}=0.349 \pm 0.032$ and $\bar{\eta}_{\text {UTlattice-incl. }}=0.400 \pm 0.028$. To investigate further this point we performed the complete UT fit either using only the exclusive value of $\left|V_{u b}\right|\left(\mathrm{All}\left[V_{u b}\right.\right.$-excl $\left.]\right)$ or only the inclusive one (All[ $V_{u b}$-incl $\left.]\right)$. In 


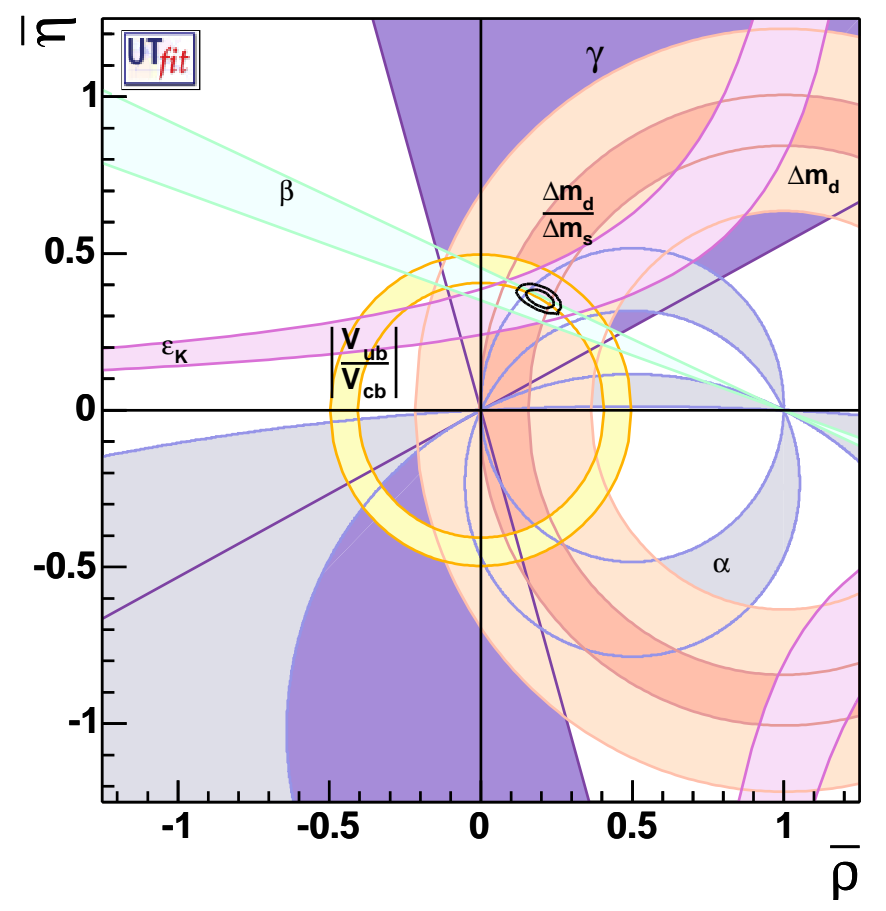

Figure 3: Determination of $\bar{\rho}$ and $\bar{\eta}$ from constraints on $\left|V_{u b}\right| /\left|V_{c b}\right|, \Delta m_{d}, \Delta m_{s}, \varepsilon_{K}, \beta, \gamma$, and $\alpha .68 \%$ and $95 \%$ total probability contours are shown, together with $95 \%$ probability regions from the individual constraints.

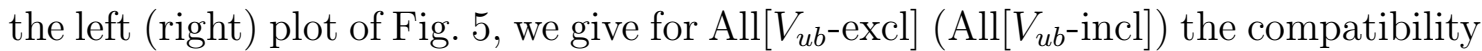
plot [9] for the inclusive (exclusive) determination of $\left|V_{u b}\right|$. We conclude that the inclusive value of $\left|V_{u b}\right|$ is not in agreement with the determination of $\left|V_{u b}\right|$ from all other constraints, at the $2.5 \sigma$ level.

- In order to investigate whether the problem originates from a tension between the experimental value of $\sin 2 \beta$ and $\left|V_{u b}\right|$, we also present the compatibility plot for $\sin 2 \beta$ including all other measurements (left plot of Fig. [6) or all other measurements except $\left|V_{u b}\right|$ (right plot of Fig. [6). We conclude that rather than a problem between $\sin 2 \beta$ and $\left|V_{u b}\right|$, the tension arises between $\left|V_{u b}\right|$ and several quantities entering the UT fit. A larger value of $\sin 2 \beta$ would only soften the problem.

- It is worth recalling that the value of $\left|V_{u b}\right|$ that is extracted from the experiments also relies on non perturbative hadronic quantities (the semileptonic form factors $f^{+}\left(q^{2}\right)$, $V\left(q^{2}\right), A_{1,2}\left(q^{2}\right)$ for exclusive $B \rightarrow \pi$ and $B \rightarrow \rho$ decays and the parameters $\bar{\Lambda}, \lambda_{1}$ and $\lambda_{2}$ for inclusive semileptonic decays). The systematic difference between the exclusive and inclusive determination of $\left|V_{u b}\right|$ (the inclusive values are always larger than the exclusive ones) might be explained by the uncertainties of the theoretical approaches. Our analysis suggests that, although all the results are still compatible, there could be some problem with the theoretical calculations, and/or with the estimate of the uncertainties, of inclusive $b \rightarrow u$ semileptonic decays. On the other hand, an effort should be made to increase the precision on the form factor of $B \rightarrow \pi$ 


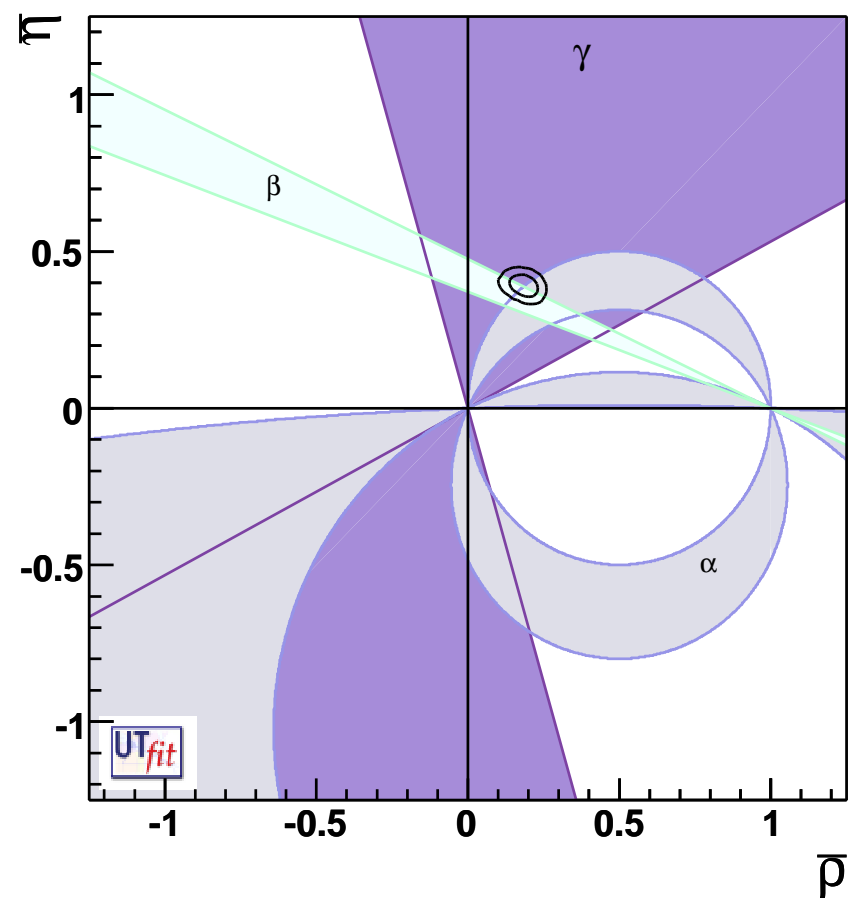

Figure 4: Determination of $\bar{\rho}$ and $\bar{\eta}$ from constraints on $\left|V_{u b}\right| /\left|V_{c b}\right|, \Delta m_{d}, \Delta m_{s}$ and $\varepsilon_{K}$ ( $68 \%$ and $95 \%$ total probability contours), compared to the $95 \%$ probability regions of the individual constraints on $\beta, \gamma$, and $\alpha$.
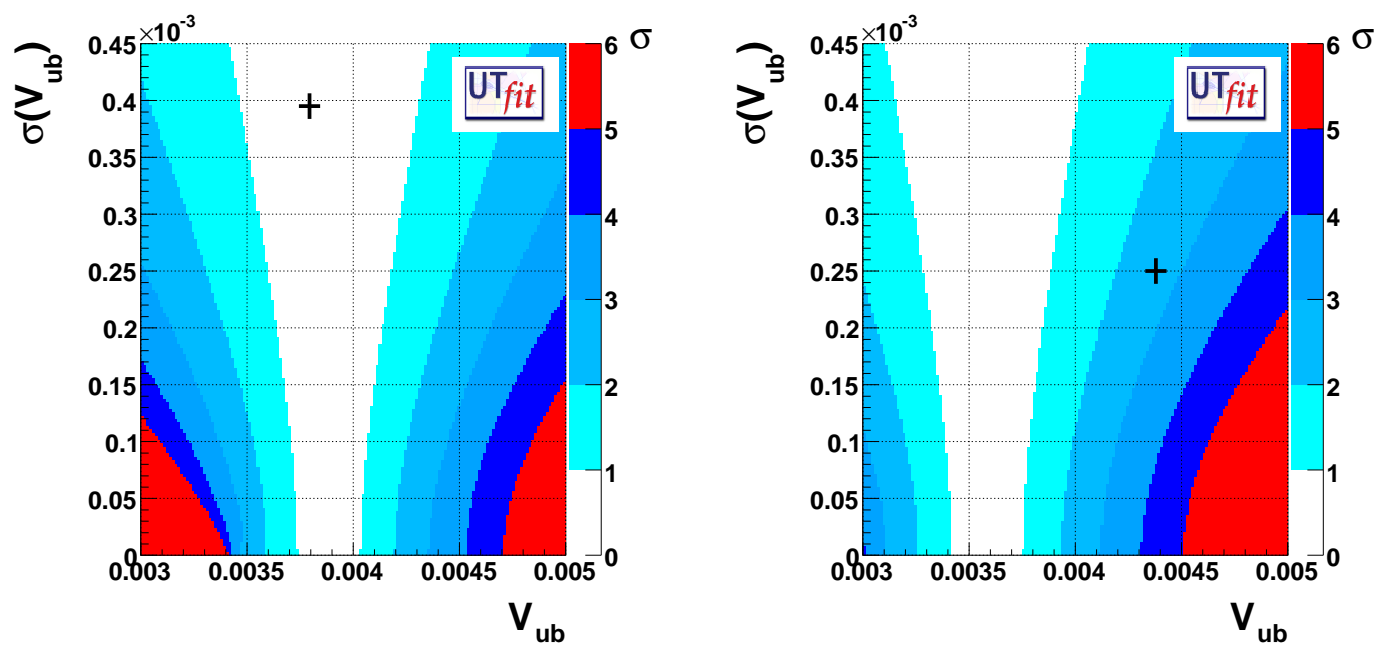

Figure 5: Left: Compatibility plot between the direct determination of $\left|V_{u b}\right|$ from exclusive analysis and the rest of the fit (including the constraint on $\left|V_{u b}\right|$ from inclusive analysis). Right:Compatibility plot between the direct determination of $\left|V_{u b}\right|$ from inclusive analysis and the rest of the fit (including the constraint on $\left|V_{u b}\right|$ from exclusive analysis). 

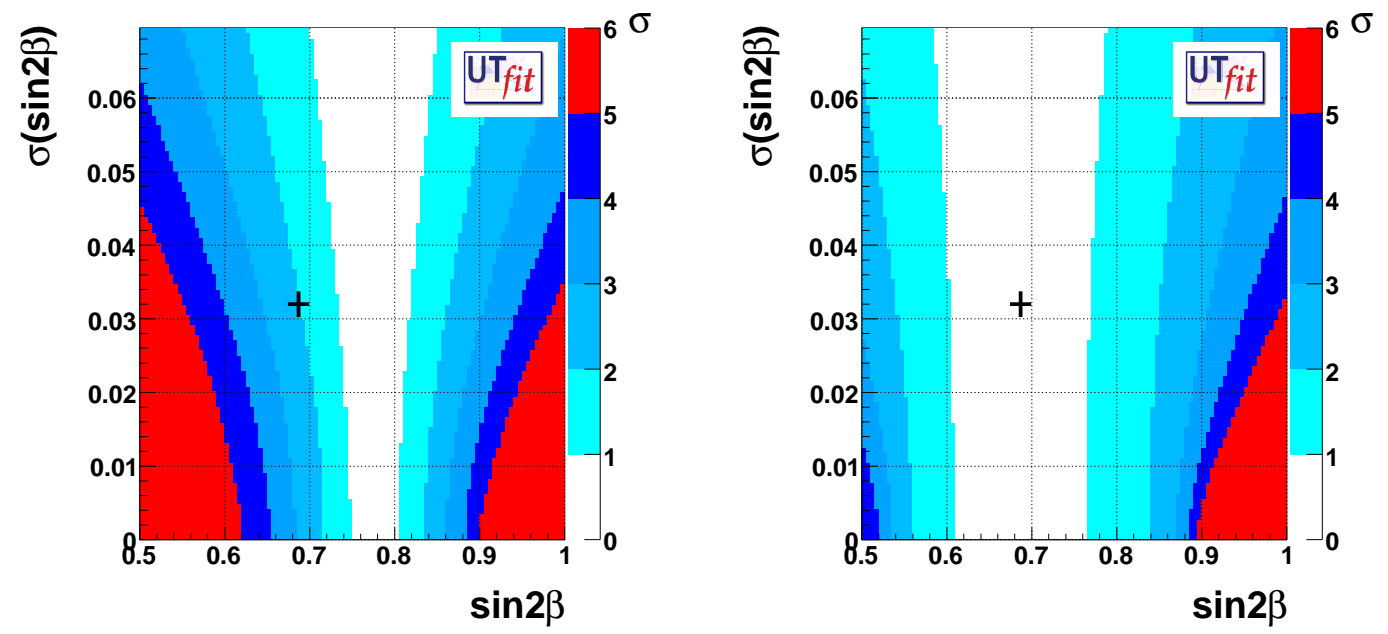

Figure 6: Compatibility plot of the experimental value of $\sin 2 \beta$ (cross) and the prediction from the fit done with all the other information, using (left) or ignoring (right) the constraint from $\left|V_{u b}\right|$.

and $B \rightarrow \rho$, providing all of them in the unquenched case, with low light quark masses and studying the continuum limit of the relevant form factors. Note that this tension among exclusive and inclusive calculations is a peculiarity of $\left|V_{u b}\right|$, since the inclusive and exclusive determinations of $\left|V_{c b}\right|$ are in much better agreement.

- Not having used $B R\left(B \rightarrow \tau \nu_{\tau}\right)$ as an input in the analysis, we can indirectly determine its value as an output of our fit. This is obtained starting from the UTangles determination of $\bar{\rho}$ and $\bar{\eta}$, combined with the experimental determination of $\left|V_{u b}\right|$ and $\left|V_{c b}\right|$, adding the experimental measurement of $\Delta m_{d}$ and $\Delta m_{s}$ to determine $f_{B} \sqrt{B_{B d}}$, and using the lattice value of $\hat{B}_{B d}, \hat{B}_{B d}=1.28 \pm 0.05 \pm 0.09$ [22] to obtain $f_{B}$ from it. In this way, the prediction is obtained without using the value of $f_{B}$ taken from lattice calculations, which has a larger relative uncertainty than $\hat{B}_{B d}$. In this way, we obtain the following values:

$$
\begin{aligned}
B R\left(B \rightarrow \tau \nu_{\tau}\right)_{\mathrm{All}} & =(1.41 \pm 0.33) \times 10^{-4} \\
B R\left(B \rightarrow \tau \nu_{\tau}\right)_{V_{u b}-\mathrm{incl}} & =(1.53 \pm 0.41) \times 10^{-4} \\
B R\left(B \rightarrow \tau \nu_{\tau}\right)_{V_{u b}-\mathrm{excl}} & =(1.02 \pm 0.22) \times 10^{-4}
\end{aligned}
$$

Although all the predictions above are compatible within the errors, a comparison of the values given in eq. (2) gives the measure of the correlation of this prediction with $\left|V_{u b}\right|$ in the overall UT fit, since all other input quantities are the same.

For comparison, with $f_{B}=(189 \pm 27) \mathrm{MeV}$ and $\left|V_{u b}\right|=(4.2 \pm 0.3) \times 10^{-3}$, one would obtain $B R\left(B \rightarrow \tau \nu_{\tau}\right)=(1.17 \pm 0.50) \times 10^{-4}$. Note that also in this case a better agreement between the prediction and the experimental world average $(B R(B \rightarrow$ $\left.\tau \nu_{\tau}\right)=(1.08 \pm 0.24) \times 10^{-4}$, combining Belle [18] and BaBar [20]) is found when the exclusive value of $\left|V_{u b}\right|$, or the value from UTangles, is used. The p.d.f. for this quantity is given in Fig. [7] 
It is important to improve the predictive power on this quantity and to clarify the situation of the $\left|V_{u b}\right|$ input, since a possible future discrepancy between the value of the experimental measurement and the theoretical prediction could signal effects of new physics from extra Higgs particles [28].

- Another possibility is to predict $\Delta m_{s}$ without using the experimental value. In order to display also in this case the correlation with the value of $\left|V_{u b}\right|$, we consider several possibilities for $\left|V_{u b}\right|$ :

$$
\begin{aligned}
\Delta m_{s}(\text { All }) & =(20.9 \pm 2.6) \mathrm{ps}^{-1} \\
\Delta m_{s}\left(V_{u b}-\text { excl }\right) & =(19.4 \pm 2.5) \mathrm{ps}^{-1} \\
\Delta m_{s}\left(V_{u b}-\text { incl }\right) & =(21.7 \pm 2.8) \mathrm{ps}^{-1}
\end{aligned}
$$

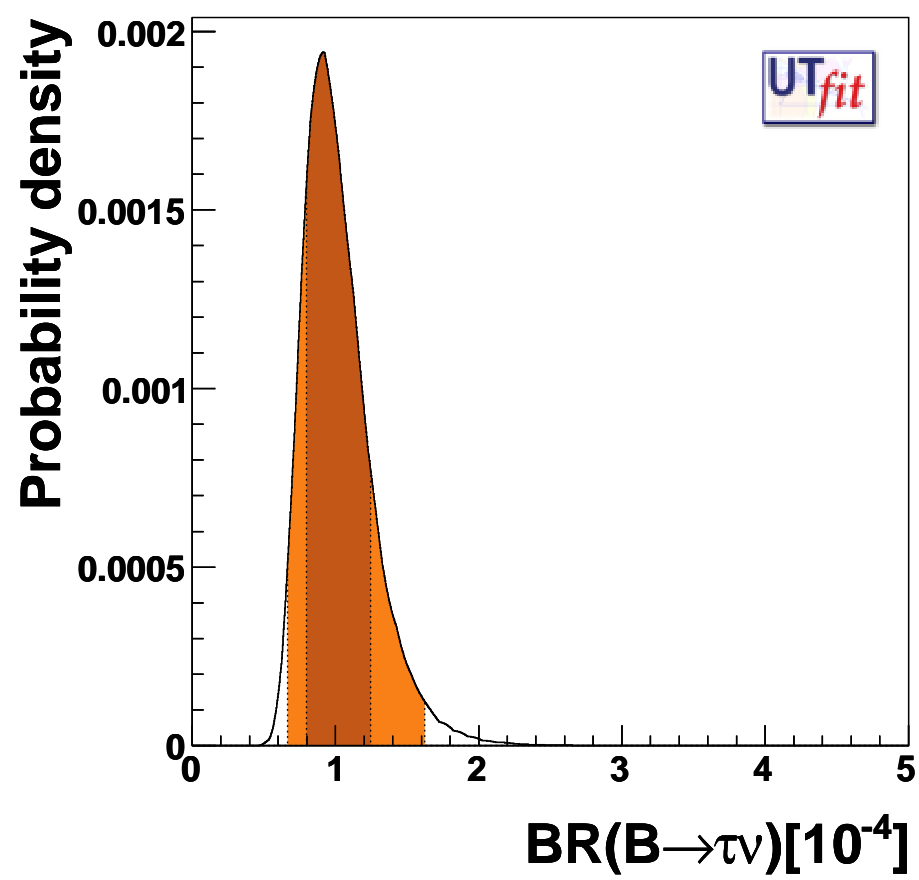

Figure 7: Determination of $B R\left(B \rightarrow \tau \nu_{\tau}\right)$ using the constraint from $\alpha, \beta, \gamma$, and $\left|V_{u b} / V_{c b}\right|$ to determine $\bar{\rho}$ and $\bar{\eta}, \Delta m_{s}$, and $\Delta m_{d}$ to fix the lattice parameters $f_{B_{s}} \sqrt{\hat{B}_{B_{s}}}$ and $\xi$, and using $\hat{B}_{B_{d}}$ from lattice $Q C D$. Only the exclusive determination of $\left|V_{u b}\right|$ is used in this case.

\section{Constraints on Lattice Parameters}

Assuming the validity of the Standard Model, the constraints in the $\bar{\rho}-\bar{\eta}$ plane from UTangles and semileptonic $B$ decay measurements, combined with the experimental values of $\Delta m_{d}, \Delta m_{s}$ and $\epsilon_{K}$, allow the "experimental" determination of several hadronic quantities which were previously taken from lattice QCD calculations. This approach has 


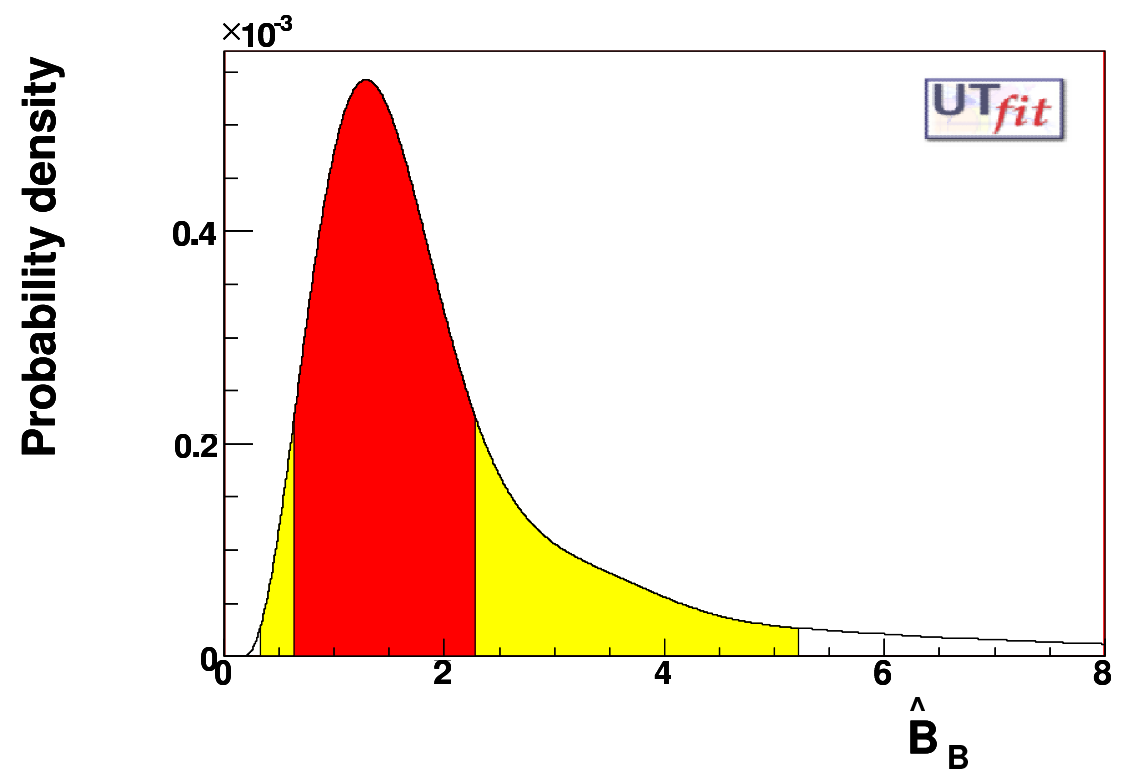

Figure 8: P.d.f. for $\hat{B}_{B_{d}}$ extracted from the UT analysis using $B R\left(B \rightarrow \tau \nu_{\tau}\right)$ to determine $f_{B}$.

two important advantages. The first one is that we have the possibility of making a full UT analysis without relying at all on theoretical calculations of hadronic matrix elements, for which there was a long debate about the treatment of values and error distributions. The second advantage is that we can extract from the combined experimental measurements the value of $\hat{B}_{K}$ and of the $B^{0}$ mixing amplitudes $f_{B_{s, d}} \hat{B}_{B_{s, d}}^{1 / 2}$ (or equivalently $f_{B_{s}} \hat{B}_{B_{s}}^{1 / 2}$ and $\xi)$ and compare them to the theoretical predictions.

Besides $\hat{B}_{K}, f_{B_{s}} \hat{B}_{B_{s}}^{1 / 2}$ and $\xi$, the measurement of $B R\left(B \rightarrow \tau \nu_{\tau}\right)$ also allows a test of the theory for the leptonic decay constant $f_{B}$, which is one of the ingredients used by lattice calculations to predict the mixing matrix element (proportional to $f_{B}^{2} \hat{B}_{B}$ ). Finally by combining the measurement of $B R\left(B \rightarrow \tau \nu_{\tau}\right)$ with $\Delta m_{d}$ and the knowledge of the angles, we can extract the value of $\hat{B}_{B_{d}}$ and compare with lattice predictions. In this case, because of the experimental error on $B R\left(B \rightarrow \tau \nu_{\tau}\right)$, we obtain a p.d.f. for $\hat{B}_{B_{d}}$ with a long tail (see Fig. (8)), corresponding to $\hat{B}_{B_{d}}=2.1 \pm 1.0,{ }^{2}$ which then is not yet competitive with the lattice prediction, $\hat{B}_{B d}=1.28 \pm 0.05 \pm 0.09$ [22]. Since the results depend on the input value for $\left|V_{u b}\right|$, we consider two cases: all the information on the UT fit is used (All) or all the information except $\left|V_{u b}\right|$ measurements, neither inclusive nor exclusive (All[no semilep]) is taken. In Tab. [3 we give the results for $\hat{B}_{K}, f_{B_{s}} \hat{B}_{B_{s}}^{1 / 2}$ and $\xi$ for these two cases. We also give the values of $f_{B}$ obtained from this fit, using in addition the lattice value of $\hat{B}_{B_{d}}$. In the last column of the table we give the lattice values for an easier comparison with those extracted from the UT fit.

We observe a better agreement with lattice calculations when $\left|V_{u b}\right|$ measurements are not included. Since the constraint provided by $\left|V_{u b}\right|$ is mainly determined by its inclusive value, in Figs. 9 we prefer to give the probability distributions for all the hadronic quantities considered in this paper $\left(\hat{B}_{K}, f_{B_{s}} \hat{B}_{B_{s}}^{1 / 2}, \xi\right.$, and $\left.f_{B}\right)$ obtained without using the

\footnotetext{
${ }^{2}$ This result is obtain using the median, which is appropriate given the long tail of the distribution. Using instead the mean we would obtain $\hat{B}_{B_{d}}=1.5 \pm 0.8$.
} 


\begin{tabular}{cccc}
\hline \hline Parameter & All & All[no semilep] & Lattice \\
\hline$\hat{B}_{K}$ & $0.94 \pm 0.17$ & $0.88 \pm 0.13$ & $0.79 \pm 0.04 \pm 0.08$ \\
\hline$f_{B_{s}} \hat{B}_{B_{s}}^{1 / 2}(\mathrm{MeV})$ & $257 \pm 6$ & $259 \pm 6$ & $262 \pm 35$ \\
\hline$\xi$ & $1.06 \pm 0.09$ & $1.13 \pm 0.08$ & $1.23 \pm 0.06$ \\
\hline$f_{B}(\mathrm{MeV})$ & $217 \pm 19$ & $202 \pm 16$ & $189 \pm 27$ \\
\hline$f_{B_{s}}(\mathrm{MeV})$ & $227 \pm 9$ & $229 \pm 9$ & $230 \pm 30$ \\
\hline \hline
\end{tabular}

Table 3: Comparison of determinations of the hadronic parameters from the constraints on the angles $\alpha, \beta$, and $\gamma$ and $\left|V_{u b}\right|$ from semileptonic decays (All) or using only the UTangles but not the semileptonic decays (All[no semilep]).

semileptonic decays, cfr. the case All[no semilep] in Tab. 3.

The value of $f_{B_{s}} \hat{B}_{B_{s}}^{1 / 2}$ from the UTfit is essentially independent of $\left|V_{u b}\right|$ and in good agreement with the lattice prediction (which has, at present, a large uncertainty). It is also interesting to extract the value of $f_{B_{s}}$ using the lattice value of $\hat{B}_{B_{s}}$, which we take equal to $\hat{B}_{B_{d}}$. Using all the constraints we obtain $f_{B_{s}}=227 \pm 9 \mathrm{MeV}$. The central value is sensibly smaller than the result predicted by the HPQCD collaboration [29], $f_{B_{s}}=259 \pm 32 \mathrm{MeV}$, although compatible within the uncertainties, and closer to other quenched or partially quenched results [22]. We believe that other unquenched calculations of the $f_{B_{s}}$, with different lattice formulations, are necessary to pin down the lattice uncertainties and make a meaningful comparison with the "experimental" number. The same holds true for $f_{B}$, for which ref. [29] quotes a value larger than many other lattice determinations. ${ }^{3}$

In Figs. 10 we show the allowed probability regions in the $f_{B_{s}} \hat{B}_{B_{s}}^{1 / 2}$ vs. $\xi$ plane, before and after the new measurement of $\Delta m_{s}$. Before having such input, we could not put an upper bound on $\xi$ since only the lower limit on $\Delta m_{s}$ was available. Now, thanks to the precision of the CDF determination, the value of $\xi$ is strongly constrained. This proves that the CDF measurement of $\Delta m_{s}$ represents a substantial progress, not only for the UT analysis, but also for our knowledge of the hadronic parameters.

The phenomenological extraction of the hadronic parameters and the comparison with lattice results assumes the validity of the SM and it is meaningful in this framework only. A similar strategy could be followed in any given extension of the SM when enough experimental information is available. In general, however, a model-independent UT analysis beyond the SM cannot be carried out without some "a priori" theoretical knowledge of the relevant hadronic parameters. For this reason the error in the calculation of the hadronic matrix elements affects the uncertainties in the determination of the NP parameters $[31,32]$.

\section{Conclusions}

The recent precise determination of $\Delta m_{s}$ by the CDF Collaboration allows a substantial improvement of the accuracy of the UT fit. Thanks to this new measurement, and to

\footnotetext{
${ }^{3}$ It is also interesting to compare the result of the fit with QCD sum rules calculations of the decay constants. For example, ref. [30] quotes $f_{B}=210 \pm 19 \mathrm{MeV}$ and $f_{B_{s}}=244 \pm 21 \mathrm{MeV}$.
} 

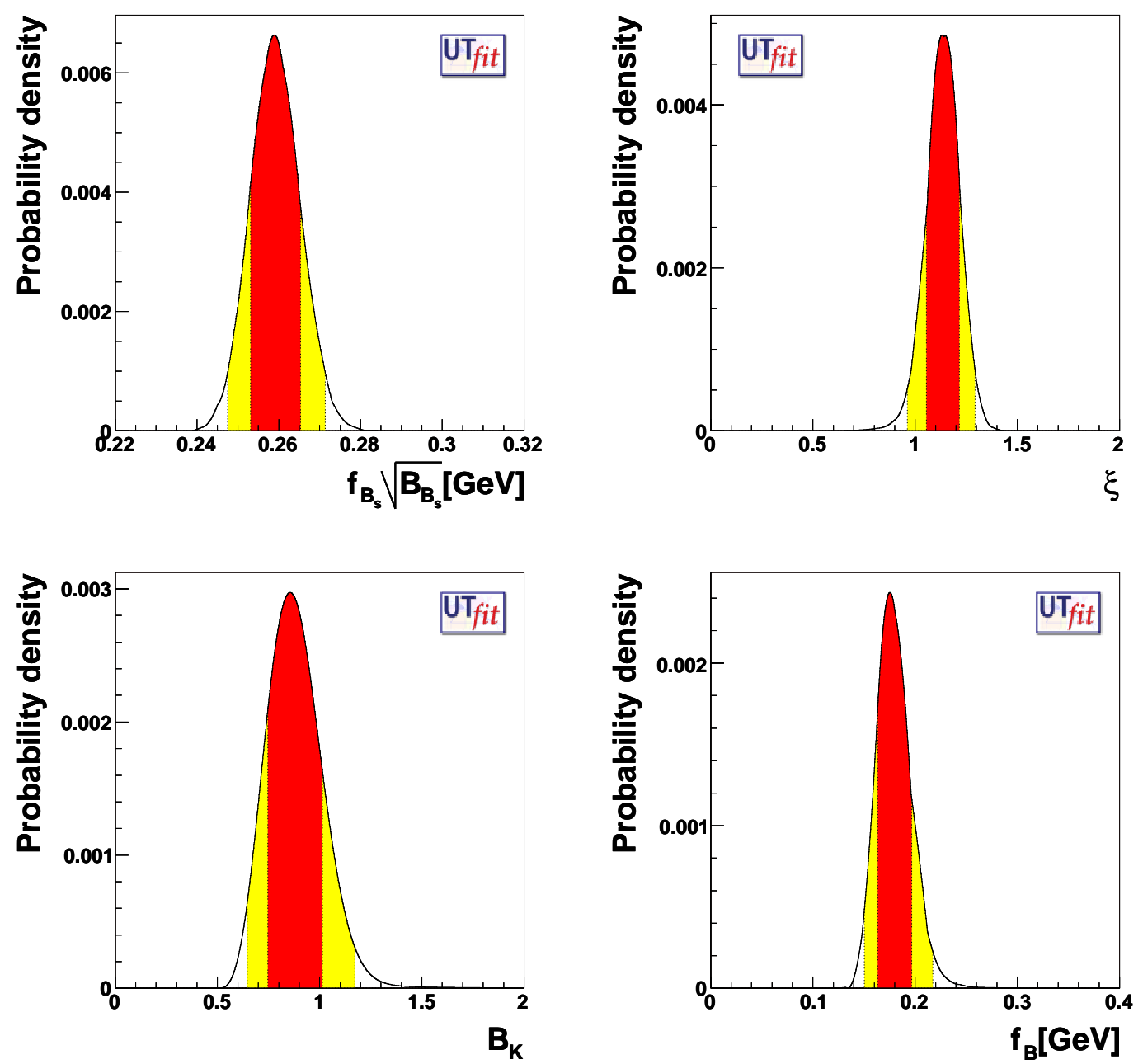

Figure 9: Determination of $f_{B_{s}} \sqrt{\hat{B}_{s}}$ (top-left), $\xi$ (top-right), $\hat{B}_{K}$ (bottom-left) and $f_{B}$ (bottom-right) obtained from the other UT constraints, using the angles information without using the semileptonic decays.

the determination of the leptonic branching fraction $B R\left(B \rightarrow \tau \nu_{\tau}\right)$ by Belle, we have shown that it is possible to extract from experiments the value of the relevant hadronic parameters, within the Standard Model. It is remarkable that the measurement of $\Delta m_{s}$, combined with all the information coming from the UT fit, allows the determination of $f_{B_{s}} \hat{B}_{B_{s}}^{1 / 2}$ with an error of $6 \mathrm{MeV}\left(f_{B_{s}} \hat{B}_{B_{s}}^{1 / 2}=257 \pm 6 \mathrm{MeV}\right)$ and of $f_{B_{s}}$ with an error of $9 \mathrm{MeV}\left(f_{B_{s}}=227 \pm 9 \mathrm{MeV}\right)$. The accuracy in the determination of $\xi$ suffers instead from the strong correlation that it has with the value and uncertainty on $\left|V_{u b}\right|$.

The only exception to the general consistency of the fit is given by the inclusive semileptonic $b \rightarrow u$ decays the analysis of which relies on the parameters of the shape function. We observed that the present determination of $\left|V_{u b}\right|$, using inclusive methods, is disfavoured by all other constraints at the $2.5 \sigma$ level. This can come either from the fact that the central value of $\left|V_{u b}\right|$ from inclusive decays is too large, or from the smallness of the estimated error, or both. Moreover the problem has been recently worsened by the decrease of the value of $\sin (2 \beta)$ determined by the direct measurements. We think that 

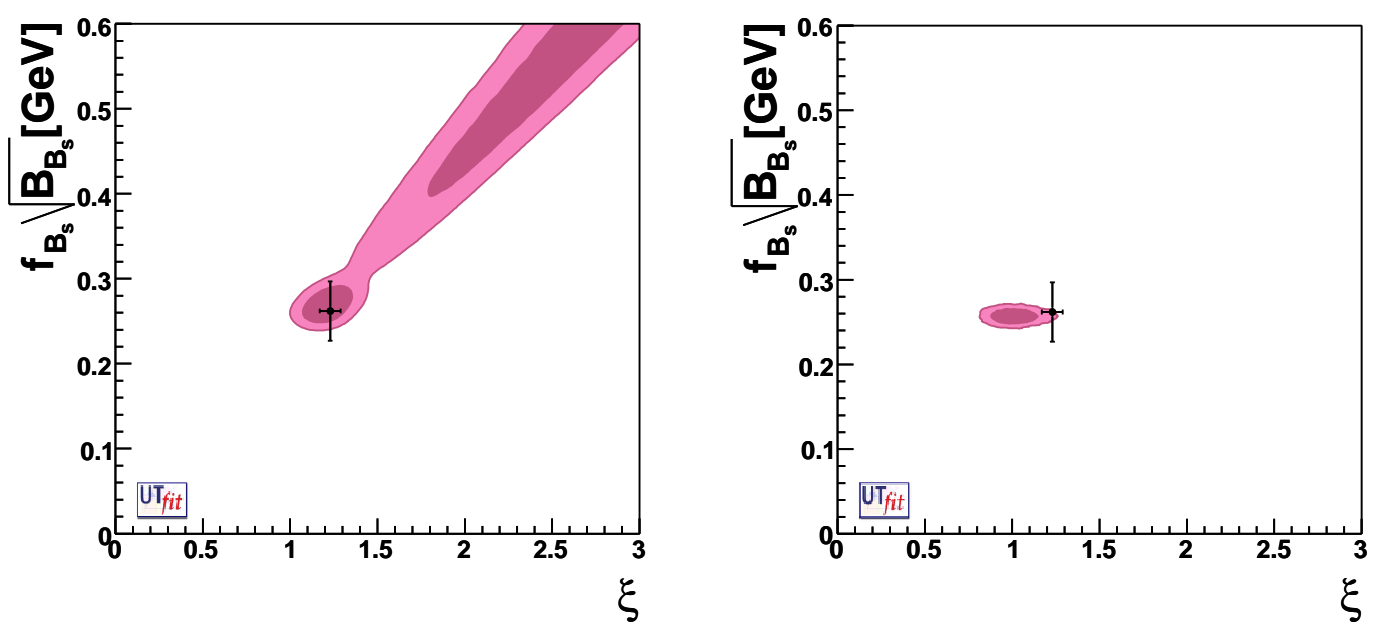

Figure 10: Constraint in the $f_{B_{s}} \sqrt{\hat{B}_{s}}$ vs. $\xi$ plane, using the UTangles result for the CKM matrix and the experimental information on $\Delta m_{d}$ and $\Delta m_{s}$. The plot on the right (left) gives the available constraint using the CDF measurement of $\Delta m_{s}$ (the upper bound before the CDF measurement). The error bars show the results from lattice QCD calculations.

it is worth investigating whether the theoretical uncertainty of the inclusive analysis has been realistically estimated.

$\left|V_{u b}\right|$ from exclusive decays has still large uncertainties and the only conclusion that we may draw is that an effort must be done for a substantial improvement of the theoretical and experimental accuracy for this quantity.

In the future, a confirmation of the results presented in this paper with smaller errors might reveal the presence of NP in the generalized UT analysis $[28,32]$. Before claiming such results, a better accuracy on the determination of $\left|V_{u b}\right|$ is however needed.

\section{Acknowledgements}

We thank G. D'Agostini and R. Faccini for informative discussions. This work has been supported in part by the EU network "The quest for unification" under the contract MRTN-CT-2004-503369.

\section{References}

[1] M. Ciuchini, E. Franco, G. Martinelli, L. Reina and L. Silvestrini, Z. Phys. C 68 (1995) 239 arXiv:hep-ph/9501265.

[2] D. E. Jaffe and S. Youssef, Comput. Phys. Commun. 101 (1997) 206 arXiv:hep-ph/9607469.

[3] P. Paganini, F. Parodi, P. Roudeau and A. Stocchi, Phys. Scripta 58 (1998) 556 arXiv:hep-ph/9711261. 
[4] M. Ciuchini et al., JHEP 0107 (2001) 013 arXiv:hep-ph/0012308.

[5] B. Aubert et al. [BABAR Collaboration], Phys. Rev. Lett. 94 (2005) 161803 arXiv:hep-ex/0408127.

[6] K. Abe et al. [Belle Collaboration], arXiv:hep-ex/0507037.

[7] A. Ali and D. London, arXiv:hep-ph/9405283; arXiv:hep-ph/9409399; Z. Phys. C 65, 431 (1995); Nuovo Cim. 109A, 957 (1996); Eur. Phys. J. C 9, 687 (1999); G. Buchalla, A. J. Buras and M. E. Lautenbacher, Rev. Mod. Phys. 68 (1996) 1125 arXiv:hep-ph/9512380. A. J. Buras and R. Fleischer, Adv. Ser. Direct. High Energy Phys. 15, 65 (1998); F. Parodi, P. Roudeau and A. Stocchi, Nuovo Cim. A 112, 833 (1999); S. Mele, in Proc. of the 5th International Symposium on Radiative Corrections (RADCOR 2000) ed. Howard E. Haber, arXiv:hep-ph/0103040; M. Bargiotti et al., La Rivista del Nuovo Cimento Vol. 23N3 (2000) 1.

[8] A. Hocker, H. Lacker, S. Laplace and F. Le Diberder, Eur. Phys. J. C 21 (2001) 225.

[9] M. Bona et al. [UTfit Collaboration], JHEP 0507 (2005) 028 arXiv:hep-ph/0501199.

[10] J. Charles et al. [CKMfitter Group], Eur. Phys. J. C 41 (2005) 1 arXiv:hep-ph/0406184.

[11] http://www-cdf.fnal.gov/physics/new/bottom/060406.blessed-Bsmix/BsMixingMeasurement.pdf

[12] M. Gronau and D. London, Phys. Rev. Lett. 65 (1990) 3381. A. E. Snyder and H. R. Quinn, Phys. Rev. D 48 (1993) 2139.

[13] M. Gronau and D. London, Phys. Lett. B253, 483 (1991); M. Gronau and D. Wyler, Phys. Lett.B265, 172 (1991); I. Dunietz, Z. Phys. C56, 129 (1992); D. Atwood, G. Eilam, M. Gronau and A. Soni, Phys. Lett. B341, 372 (1995); D. Atwood, I. Dunietz and A. Soni, Phys. Rev. Lett. 78, 3257 (1997); D. Atwood, I. Dunietz and A. Soni, Phys. Rev. D 63 (2001) 036005; A. Giri, Yu. Grossman, A. Soffer and J. Zupan, Phys. Rev. D68, 054018 (2003).

[14] I. Dunietz, Phys. Lett. B 427 (1998) 179 arXiv:hep-ph/9712401.

[15] I. Dunietz, H. R. Quinn, A. Snyder, W. Toki and H. J. Lipkin, Phys. Rev. D 43 (1991) 2193. J. Charles, A. Le Yaouanc, L. Oliver, O. Pene and J. C. Raynal, Phys. Rev. D 58 (1998) 114021 arXiv:hep-ph/9806347|. A. S. Dighe, I. Dunietz and R. Fleischer, Phys. Lett. B 433 (1998) 147 |arXiv:hep-ph/9804254|.

[16] I. Dunietz and R. G. Sachs, Phys. Rev. D 37 (1988) 3186 [Erratum-ibid. D 39 (1989) 3515].

[17] I. I. Y. Bigi and A. I. Sanda, Nucl. Phys. B 193 (1981) 85. For a recent estimation of the theoretical error, see M. Ciuchini, M. Pierini and L. Silvestrini, Phys. Rev. Lett. 95, 221804 (2005) arXiv:hep-ph/0507290|.

[18] K. Ikado et al., [Belle Collaboration], arXiv:hep-ex/0604018.

[19] V. Abazov et al. [D0 Collaboration], arXiv:hep-ex/0603029 
[20] B. Aubert et al. [BABAR Collaboration], Phys. Rev. D 73 (2006) 057101 arXiv:hep-ex/0507069.

[21] C. Dawson, PoS LAT2005 (2005) 007.

[22] S. Hashimoto, Int. J. Mod. Phys. A 20 (2005) 5133 arXiv:hep-ph/0411126.

[23] E. Barberio et al. [H. F. A. Group], arXiv:hep-ex/0603003.

[24] http://www.utfit.org

[25] J. Charles, arXiv:hep-ph/0606046.

[26] M. Battaglia et al., Based on the First Workshop on "CKM Unitarity Triangle" held at CERN, 13-16 February 2002. Edited by: M. Battaglia, A. Buras, P. Gambino and A. Stocchi. CERN-2003-002-corr 10 October 2003, arXiv:hep-ph/0304132.

[27] Y. Nir, arXiv:hep-ph/0510413.

[28] W. S. Hou, Phys. Rev. D 48 (1993) 2342; G. Isidori and P. Paradisi, arXiv:hep-ph/0605012,

[29] A. Gray et al. [HPQCD Collaboration], Phys. Rev. Lett. 95 (2005) 212001 arXiv:hep-lat/0507015.

[30] M. Jamin and B. O. Lange, Phys. Rev. D 65 (2002) 056005 arXiv:hep-ph/0108135.

[31] M. Bona et al. [UTfit Collaboration], JHEP 0603 (2006) 080 arXiv:hep-ph/0509219.

[32] M. Bona et al. [UTfit Collaboration], arXiv:hep-ph/0605213. 